\title{
THE PATHOLOGY OF EPIDEMIC PNEUMONIA IN
}

MICE AND GUINEA-PIGS *

\section{J. JAY KEEGAN, M.D. \\ BOSTON}

The subject of epidemic pneumonia has been receiving a great amount of attention since the recent epidemics of streptococcus pneumonia and influenzal pneumonia. There are still many unknown factors of pathogenicity, virulence, invasiveness and toxicity of microorganisms which produce epidemic diseases. These terms commonly are used without clear distinction of meaning. ${ }^{1}$ The difficulty of reproducing characteristic epidemic lesions or even causing infection in animals with cultures of highly specialized strains of bacteria is well known. In view of these difficulties in experimental reproduction of an epidemic disease, it has seemed possibly more profitable to study the natural processes of an animal epidemic of pneumonia, followed through its entire course of development and subsidence.

The opportunity to observe and study such an epidemic presented itself in the animal room of the U. S. Naval Hospital, Chelsea, Mass., during the late winter and early spring of 1919 . Distemper and epidemic pneumonia in laboratory animals has been the subject of several valuable papers, ${ }^{2}$ but these investigations have been directed primarily toward establishing the etiologic relationship of the Bacillus bronchicepticus to these diseases. Very good evidence has been presented that this bacillus is the causative micro-organism in certain common epidemics of distemper and pneumonia.

In order to interpret properly the origin and course of this animal epidemic, it is necessary to describe the location of the cages in the animal room and give the number of animals exposed to the infection. This room measured ten feet square and had three steel shelves for cages on each of two opposite sides. On one side there were only guinea-pig cages, three rows of four each. On the other side the two lower shelves held eight guinea-pig cages and the top shelf twelve mice cages. At the beginning of the epidemic there were about fifty guinea-pigs in stock. During the epidemic, three lots of a dozen each were received from outside stock, making a total of eighty-six pigs exposed to the infection. Of these it is estimated that twenty-five

* From the U. S. Navy and the Surgical Clinic, Peter Bent Brigham Hospital. Hospital.

1. Topley, W. W. C.: The spread of bacterial infection, Lancet 1:1, 1919.

2. Ferry, N. S.: A preliminary report of the bacterial findings in canine distemper, Am. Vet. Rev. 37:499, 1910. 
developed recognizable symptoms of respiratory disease; a necropsy was performed on fifteen. The mice cages were crowded together, partly placed on top of one another, contained more mice than intended for their size, and were harder to keep clean than the guinea-pig cages. There were about 150 mice in these cages at the beginning and they were being increased rather rapidly by breeding. These factors probably favored the origin of the infection in the mice. Twenty-four mice with symptoms of respiratory infection were later subjected to necropsy. Several died during the early part of the epidemic and were not examined. Many others were noted with distinct or questionable illness and recovered. A conservative estimate of the total number observed sick would be fifty.

The infection in the mice at first had the characteristics of what is commonly called distemper in animals. The sick mice were identified by the affected appearance of their eyes, there being an excessive conjunctival secretion, somewhat purulent, with swollen partly closed lids and desquamation and depilation in the surrounding epithelium. Occasionally a nasal discharge and cough were noted. There also was some indisposition and roughening of the fur. Few died during this period and bacteriologic or pathologic examinations were not made. It seemed very probable, however, from these observations and the subsequent course, that the epidemic pneumonia took origin in this manner.

The first intimation of a bronchial infection among the mice occurred a few weeks after the distemper symptoms had been noted. There had been a continuation and increase of the distemper symptoms when it was observed that a number of the mice had distinctly abnormal respirations. The breathing was more rapid than normal, but most noticeable was its deep or labored character, with heaving sides and use of accessory muscles of respiration, the effort fairly shaking the animal. These mice otherwise did not appear particularly sick. Their fur was slightly roughened and they were gaunt, but they were quite active when disturbed and were feeding. Some of these mice were isolated for a time, did not die within a week or two, so were killed and examined.

The necropsies presented constantly a firm grayish white lobular consolidation in one or more lobes, most frequently the median and cephalic lobes, rarely the larger caudal lobes which usually were the seat of a compensatory emphysema. There was no pleurisy. On gross section the dilated bronchi could be seen distinctly and a thick purulent exudate expressed from the lumen. The walls of the bronchi were thickened by a firm grayish infiltration. The smaller lobes were completely involved. The larger lobes, particularly the large 
caudal lobes, frequently presented a similar grayish peribronchial infiltration along the main bronchi near the hilus, the remainder of the lobe being emphysematous.

Microscopic examination of these lungs disclosed excellent examples of purulent bronchitis, or interstitial bronchopneumonia as defined by $\mathrm{MacCallum}^{3}$ in postmeasles streptococcus bronchopneumonia. Various stages were found. Typically the bronchi were dilated and filled with a dense polymorphonuclear exudate (Fig. 1). The mucosa tended to be a thick hyperplastic layer with very thick and long cilia, but in some places was desquamated. The bronchial walls were greatly thickened by a dense infiltration of mononuclear cells of the lymphoid variety. The thickness varied with the duration and stage of the disease and the lobe examined. A striking feature in many sections was the mononuclear cell infiltration about blood vessels, of similar character to the peribronchial infiltration.

The terminal bronchioles in these cases frequently appeared to drain areas of dense polymorphonuclear alveolar exudate. It seemed possible that the main part of the polymorphonuclear exudate found farther along in the dilated bronchi might come from these alveolar lesions, the irritant features of which would give rise to the more chronic protective mononuclear peribronchial reaction. In many places, however, a distinct passage of polymorphonuclear cells through the bronchial mucosa could be seen.

The alveoli of these lungs presented variable conditions, in addition to the focal polymorphonuclear lesion at the terminal bronchioles. There was commonly a partial or complete atelectasis of the alveoli intervening between enlarged bronchi. The exudate within these alveoli consisted chiefly of sparse mononuclear cells, some containing pigment granules, an occasional polymorphonuclear leukocyte or finely granular albuminous material. In lobes in which there was only partial hilus bronchial involvement, the alveoli of adjacent uninvolved areas were markedly emphysematous.

Such cases of purulent bronchitis continued to occur among the mice for a few weeks, with occasional deaths after an illness of several days or weeks. Most of the distinctly sick mice were killed at selected periods and examined. As the infection progressed the areas of polymorphonuclear alveolar exudate increased and the mononuclear peribronchial infiltration decreased. Rather suddenly it was noticed that the type of the disease had changed. Instead of the prolonged illness with marked respiratory symptoms and few toxic symptoms, mice were found dead in cages where routine inspection

3. MacCallum, W. G.: Pathology of pneumonia at a base hospital, J. A. M. A. 70:1150 (April 20) 1918. 
on the preceding day had not shown any affected mice. Closer inspection on succeeding days identified these fulminating cases by their inactiveness and their slow and somewhat irregular respirations.

Necropsies done on these mice with toxic symptoms and fulminating course, found clead or killed, presented constantly hemorrhagic lesions in the lungs. The hemorrhagic areas varied from a light red, mottled with air filled alveoli, to a solid very clark red color. The gross appearance was striking. The smaller and more dependent lobes

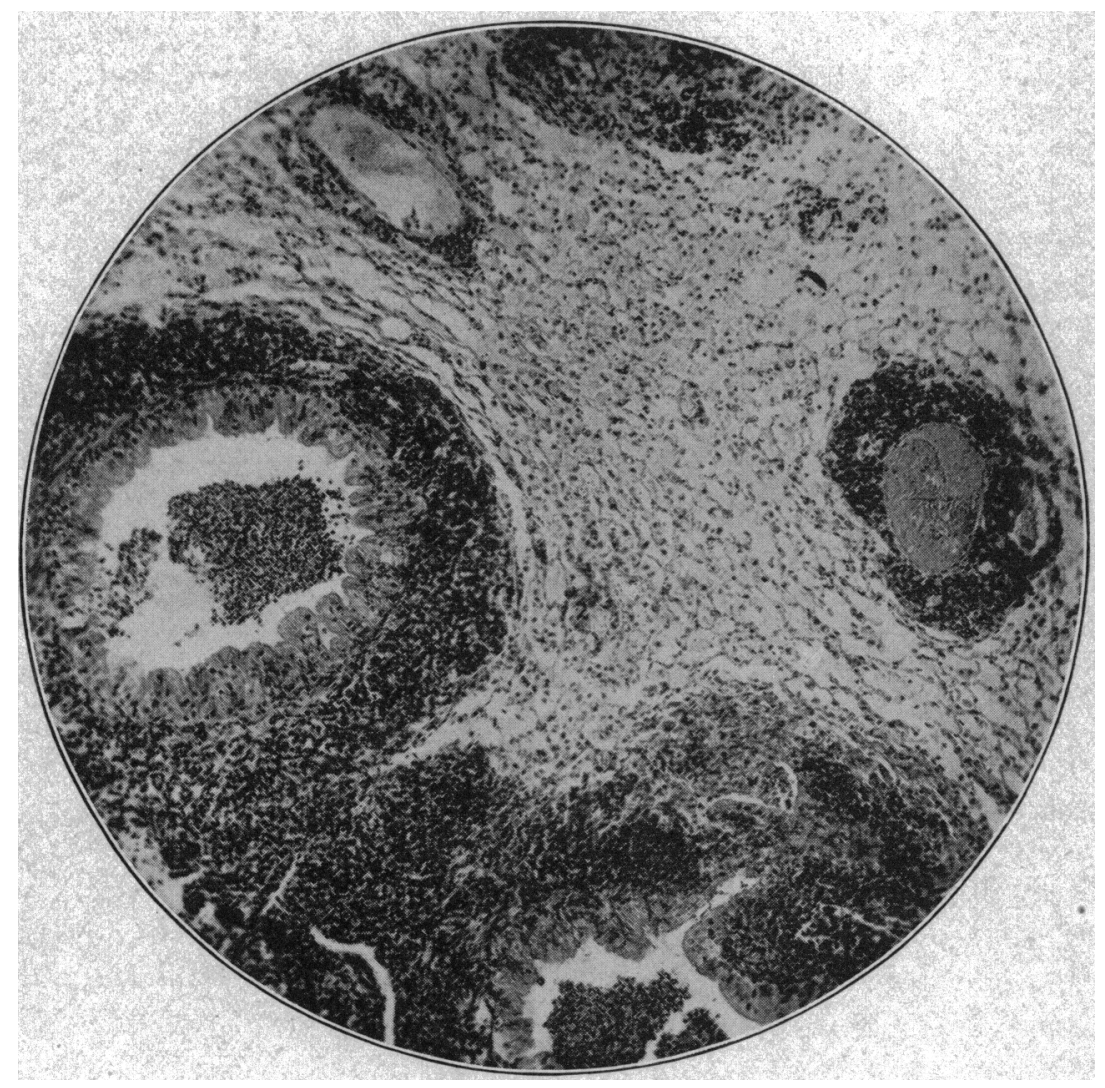

Fig. 1.-Microphotograph of a lung section from mouse 1, showing an advanced active purulent bronchitis. The bronchi are filled with polymorphonuclear leukocytes, the mucosa is hyperplastic and the wall densely infiltrated with mononuclear cells. There is a similar mononuclear perivascular infiltration. The alveoli are atelectatic, containing serum and a few mononuclear cells. Cultures gave a pure growth of the Bacillus bronchisepticus.

were most affected, the caudal lobes commonly being distinctly emphysematous and pink. Lobes which presented only a slight red mottling on the surface contained more extensive hemorrhagic areas in the center. 
Microscopic sections of these hemorrhagic lungs presented a type of pneumonia rarely met, except in fulminating cases at the height of an epidemic of pneumonia. It is rare probably because it is a stage much earlier than is seen at necropsies of pneumonia of pneumococcus origin, or of low grade pneumonias of other origin. The lesion is a rather irregular central or lobular alveolar hemorrhage with numerous distended air spaces scattered thronghout the area (Fig. 2).

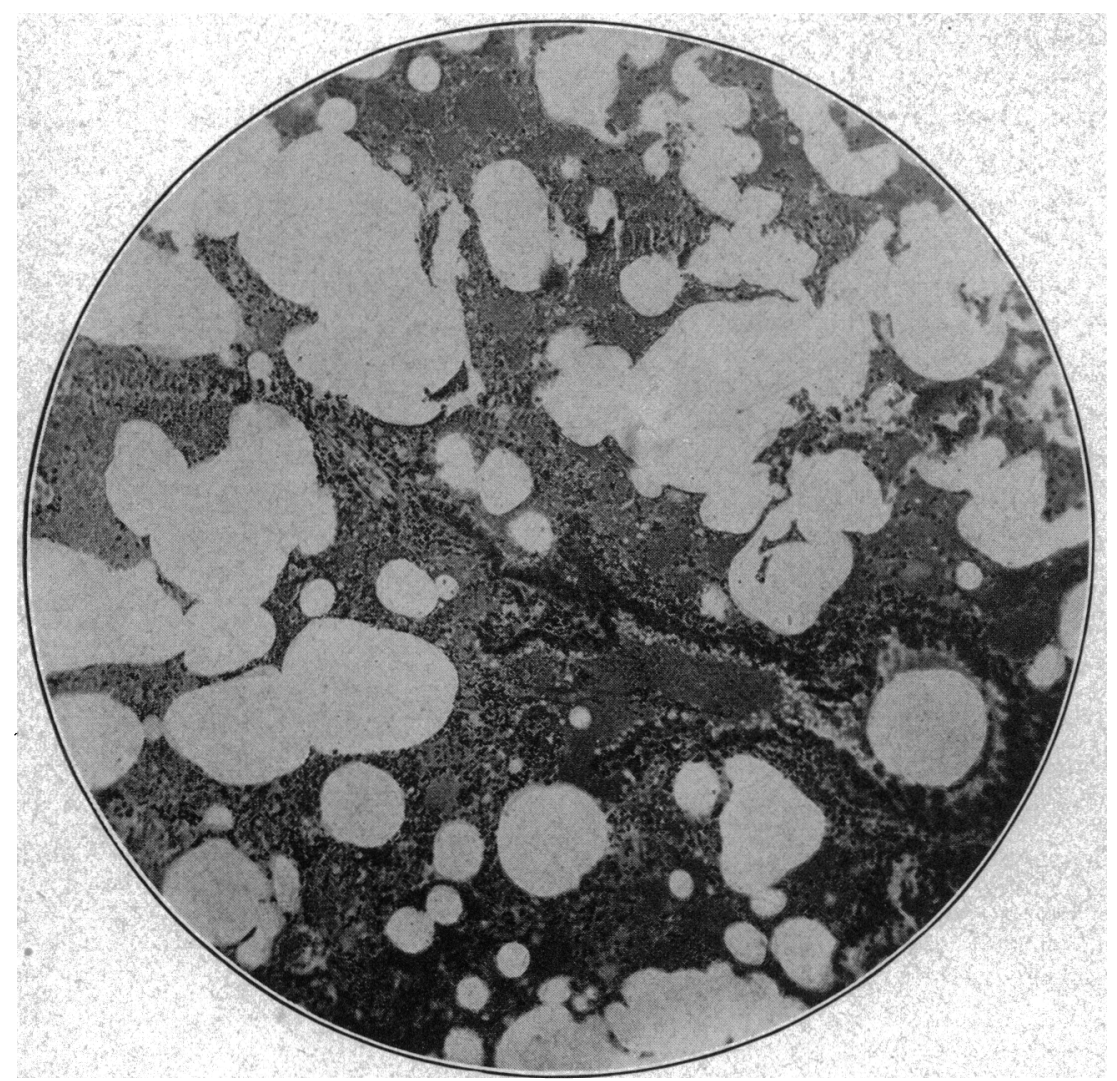

Fig. 2.-Microphotograph of a lung section from mouse 23, showing an emphysematous hemorrhagic type of pneumonia characteristic of the acute toxic stage of the epidemic.

Various stages of this were found. Some mice died at a very early period when no other lesion than an extreme capillary engorgement and serous alveolar exudation could be found, although usually in the central part of the lobes areas of alveolar hemorrhage were present.

The subsidence of the epidemic among the mice was prolonged and marked by a recrudescence of the acute toxic hemorrhagic form of the disease. Greater numbers of the sick mice began to have promi- 
nent respiratory embarrassment and prolonged course. Such mice at necropsy presented a great variety of lesions in different lobes or even in the same lobe; purulent bronchitis in many stages of heal. ing with obliteration of bronchi, limited or widespread polymorphonuclear alveolar exudate, lobular or fused areas of alveolar hemorrhage or simple capillary engorgement.

The infection among the guinea-pigs appeared later than among the mice and apparently took origin from the prevailing mouse epidemic at about the height of the latter. It is recalled that there were two rows of guinea-pig cages below the mice cages on one side of the animal room. The first infected guinea-pigs were noted in the row directly beneath the mice, and the infection was limited to the pigs of this side of the room for two weeks. The spread to the other side of the room dated from a general cleaning and sterilization of the cages, isolation of all sick pigs and transfer of all healthy appearing pigs to common outdoor pens or to cleaned cages on the uninfected side of the room. This attempt to rid the animal room of all infected cages only served to spread the disease. This was understood later when it was found by many necropsies and cultures that in an infected stock many apparently normal pigs show some lung lesion and give positive cultures of the Bacillus bronchisepticus from the trachea in more than 50 per cent.

\section{PROTOCOLS}

The symptoms of the first guinea-pig affected were those of a very severe primary toxic reaction with early appearance of marked respiratory embarrassment. The following is the protocol:

PIg 21: History.-A $300 \mathrm{gm}$. animal in a cage with six other pigs, directly beneath infected mice cages.

April 4, 1919: Breathing rather rapidly, with occasional pause and expiratory grunt; inactive; fur roughened; evidently sick.

April 5: At 2 p. m., respirations 200 per minute, labored, almost constant expiratory grunt; occasional cough; pig inactive. Leukocyte count, 11,300; polymorphonuclears, 65 per cent.; mononuclears, 35 per cent.; temperature, 101.8 F. At 6 p. m., respirations 180 . At 12 p. m., pig appeared somewhat better, respirations still rapid and labored, however.

April 6: At 3:30 a. m., and at 7 a. m., condition unchanged; still quite rapid respirations with grunting expiration, inactive, fur roughened, but not appearing quite so sick as on previous day. At $1: 30$ p. m., respirations 170; temperature $100.4 \mathrm{~F}$., evidently improving. At $3 \mathrm{p}$. m., killed by blow on back of head.

Necropsy.-There was a slight increase of peritoneal fluid; serous surfaces smooth and glistening; no evident pathology in abdomen. The medial, ventral and cephalic lobes of the left lung appeared completely involved in a firm lobular gray consolidation. A similar type of involvement was present in the same lobes on the right side but chiefly central in the cephalic lobe. Both caudal lobes were large, soft and crepitant, appearing normal, except for 
moderate emplysema. The cut surface of these lobes, however. disclosed a few small gray areas near the hilus along the main bronchi, and a few punctate hemorrhages in the remainder of the lobe.

Microscopic: Sections revealed a fairly uniform type of pathology with varying degrees of involvement. The gray nodules seen in gross were areas of dense polymorphonuclear alreolar exudate, localized very definitely in the alveoli surrounding terminal bronchioles (Fig. 3). This lesion apparently began at the point of transition of the cuboidal epithelium of the terminal bronchiole to the flat epithelium lining the alveoli of the infundibulum. The infundibulum and surrounding alveoli are seen packed with polymorphonuclears

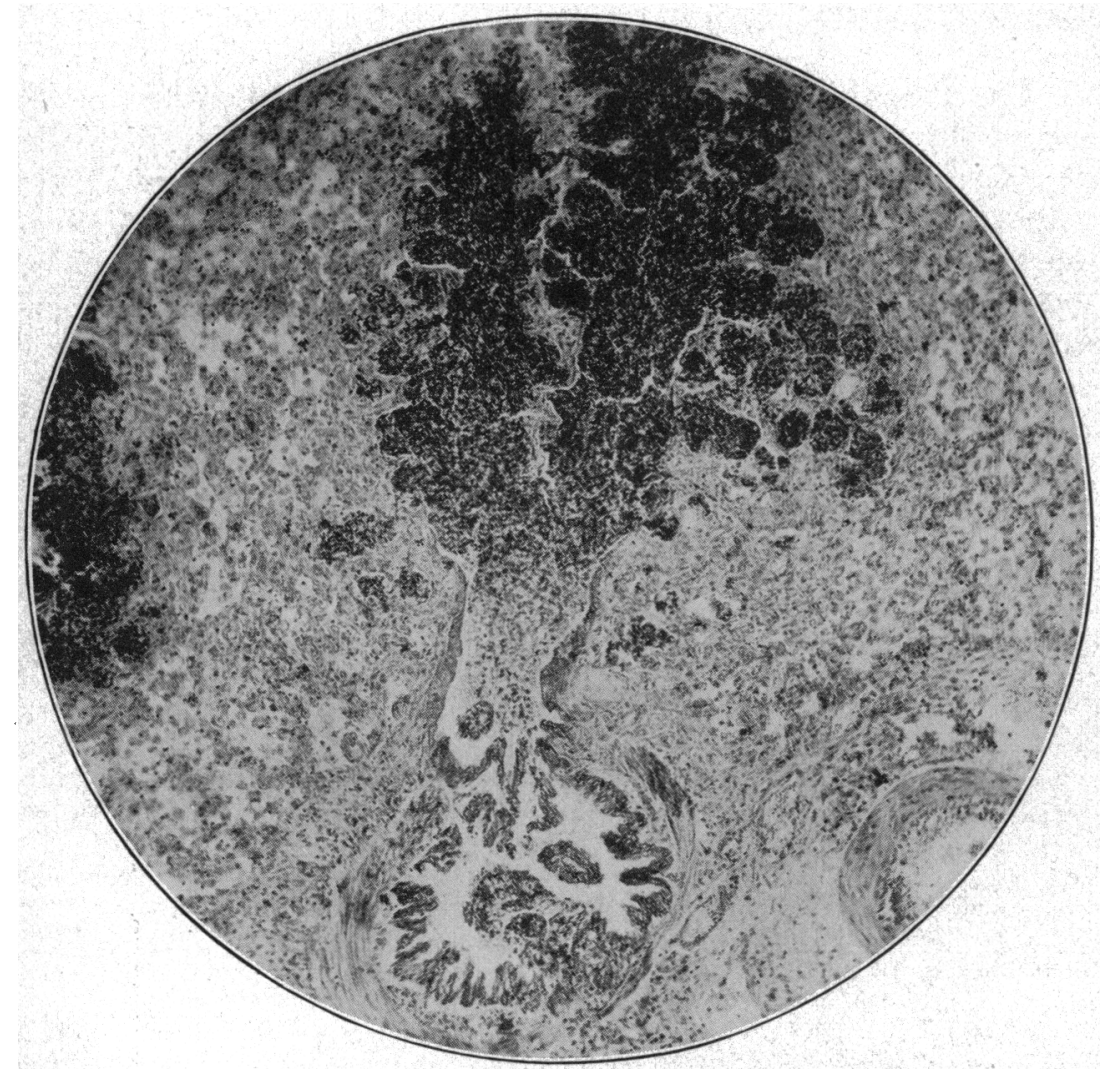

Fig. 3.-Microphotograph of a lung section from guinea-pig 21, showing an ddvanced infundibular lesion. This consists of an area of dense polymorphonuclear alveolar exulate subtended by a terminal bronchiole. The active process is distinctly delimited by the line of transition from the cuboidal epithelium of the terminal bronchiole to the flat epithelium of the infundibulum. There is no reaction about the bronchus except the general atelectasis and consequent slight cellular infiltration. The bronchial lumen contains only degenerated leukocytes which appear to have drained from the infundibulum.

which have not extended in great numbers into the bronchus but which evidently have blocked the terminal bronchiole. The alveoli beyond this rather sharply localized polymorphonuclear reaction are atelectatic, having a narrowed lumen, enlarged epithelial cells and a content of granular material with a few 
large mononuclear cells. The small focal hemorrhages seen in gross section of the caudal lobes are identified as similarly localized areas of infundibular and surrounding alveolar hemorrhage, with blocking of the terminal bronchiole and inclusion of air bubbles in the hemorrhagic exudate. The best examples of this are found surrounding terminal bronchioles near the main bronchus of the lobe, indicating that the progress of the infection is by direct continuity of the bronchial mucosa, the later infection in the caudal lobes probably being controlled to some extent by gravity and distance.

Bacteriologic: A heavy growth of the Bacillus bronchisepticus was obtained from the trachea and the left cephalic lobe of the lung. Cultures from the heart's blood, peritoneum, pericardium and right caudal lobe of lung were negative. No other micro-organisms were present.

The following day, April 7, another guinea-pig on the same side of the room was isolated on account of ill defined symptoms of illness. The respirations were not increased, were quite shallow and only slightly irregular. This pig was under constant observation during the afternoon and there was some question from the symptoms whether he was sick or not. Unfortunately, he was killed late that night and no tissues were saved. The gross description of the organs at necropsy, however, gave a diffuse emphysematous hemorrhagic lesion in all lobes of the lungs as the only evident pathology. Cultures were not made.

April 8, a pig was found dead in a cage beneath the mice cages. This cage had been inspected on the previous day and this pig had not been identified as sick, although most careful observations were being made at this time. The protocol follows:

PIg 22: History.-April 8, 1919, found dead, evidently only a short time postmortem. Blood tinged froth was seen in the nostrils.

Necropsy.-Abdomen negative, except moderate vascular injection of viscera. The lungs were voluminous, not collapsing on opening the thorax, and there was an increased amount of clear fluid in both pleural cavities. The left cephalic lobe was mottled with dark red, chiefly central, and pink emphysematous areas, chiefly peripheral. The ventral lobe was a dark red color throughout and more firm. The left caudal lobe was more nearly normal but voluminous and contained scattered small focal areas of apparent hemorrhage. The lobes of the right lung were similar but less involved.

Microscopic: The predominant lesion in all lobes was a marked capillary engorgement with variable serous exudation in the alveoli. The central or hilus portions of the lobes gave evidence of a more severe capillary lesion. In many places there was thrombosis of the capillaries with evident degeneration of the alveolar septums, desquamation of the epithelial cells, beginning infiltration with polymorphonuclear leukocytes and serous exudation into the alveoli (Fig. 4). Alveolar hemorrhage was not prominent. The localization of these areas of most severe involvement did not throw much light on their manner of origin, whether in relation to bronchi or bloodvessels. There was an extreme edema of the perivascular tissue surrounding the larger blood vessels. The bronchi contained an eosin staining coarse granular material. The terminal bronchioles did not show a greater reaction than other regions and did not appear to be blocked by exudate.

Bacteriologic: Lung cultures gave a heavy pure growth of the Bacillus bronchisepticus. Sections stained for bacteria with Giemsa's stain demonstrated large numbers of small bacilli in the bronchi and in the more involved regions. In the bronchi they were located along the epithelial border but could 
not be found, except rarely, in the bronchial wall. Immediately surrounding the larger bronchi they were especially abundant in the alveoli and the alveolar walls. There appeared to be a similar concentration about the larger veins. The areas of capillary thrombosis always contained many bacilli, not in the thrombosed capillaries but in clumps or within cells in the alveoli or in the alveolar walls in regions of degeneration.

It was at about this time that an attempt was made to check the epidemic by rigid isolation of sick pigs and sterilization of cages,

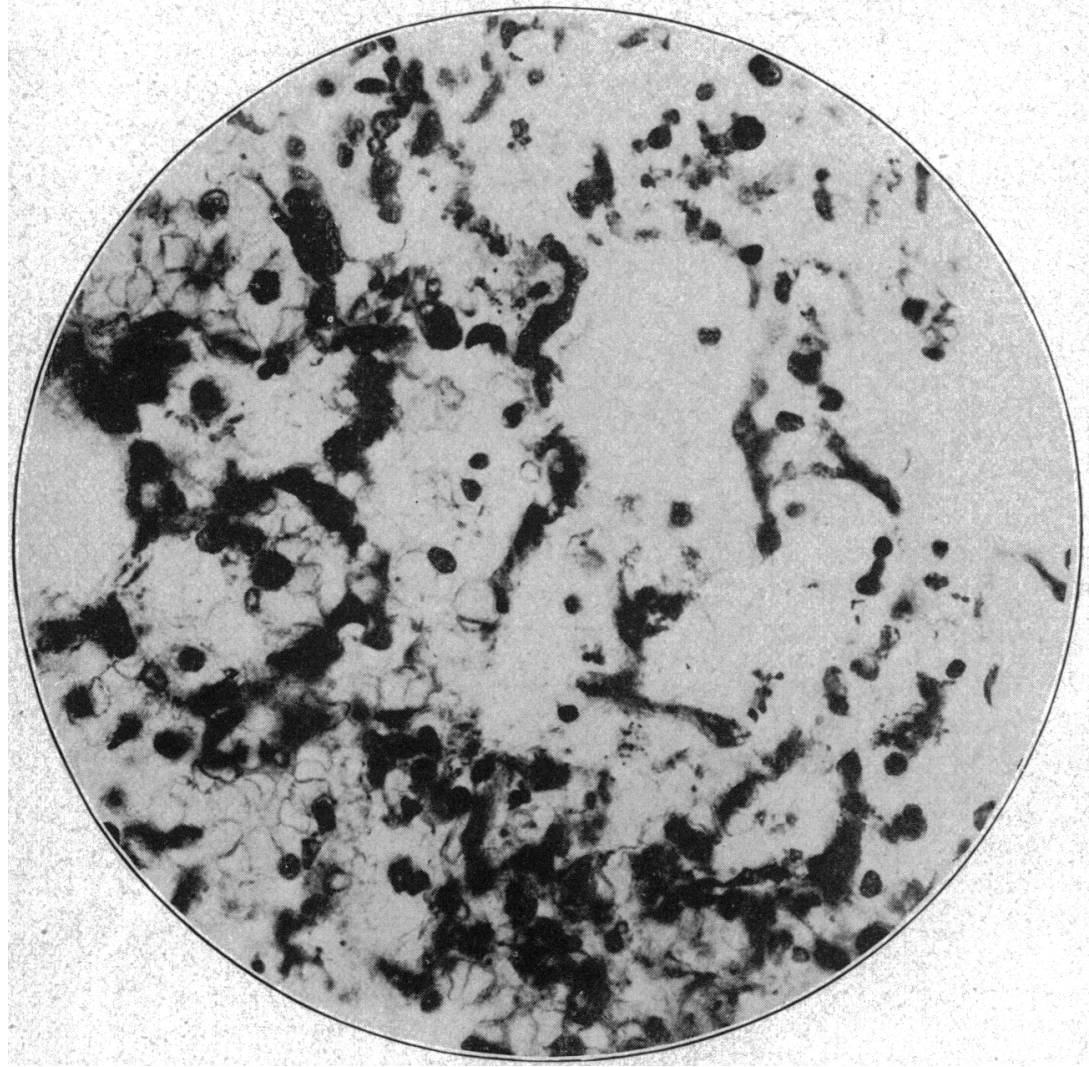

Fig. 4.-Microphotograph of an area of capillary thrombosis in the lung of guinea-pig 22. The thrombi are indicated by the irregular branching darkly stained areas. There is considerable necrosis of the alveolar walls, serous exudation and beginning polymorphonuclear infiltration. Capillary engorgement and hemorrhage are evident at the left side.

with transfer of some of the normal appearing pigs to common outside pens. But pigs continued to become sick, now in the outside pens and on the other side of the animal room. The following protocol is that af a breeding female from the outside pen and illustrates a pneumococcus complication: 
PIg 25: History.-April 12, 1919, noted inactive and not feeding. That night a miscarriage occurred. The respirations increased moderately during the next two days, the pig becoming gaunt and weak. Killed April 15.

Vecropsy.-There was found a massive gray lobular consolidation of both cephalic lobes and a more uniform darker consolidation of the ventral and median lobes. The caudal lobes were emphysematous, did not collapse and presented only small nodules along the main bronchi.

Microscopic: Sections presented a type of pathology somewhat different from that previously found. The predominant lesion was a rather widespread polymorphonuclear alveolar exudate, distinctly lobular in its distribution, yet confluent in many places. Very little fibrin was present, although places were found in which there was the typical fibrinopurulent alveolar exudate of pneumococcus pathology. The most characteristic lesion found in this and other pigs with a secondary pneumococcus infection was focal necrosis of variable size and shape.

Bacteriologic: Lung cultures from the ventral lobes gave a pure growth of the Bacillus bronchisepticus. A pneumococcus, tested by inulin fermentation and bile solubility, was recovered from the left median lobe.

The following protocol represents an infection in a newly received group of guinea-pigs, another instance of which is Pig 40 . There seemed to be a tendency for acute cases to occur in new stock a few days after their receipt.

PIG 26: History.-April 15, noted somewhat inactive, yet of questionable illness. The following morning he was distinctly ill, with roughened fur, rather deep but not increased respirations, and noted occasionally coughing and rubbing his nose. At $5 \mathrm{p}$. m. April 16, respirations were 100 per minute; temperature, $101.6 \mathrm{~F}$.; leukocyte count, 8,500 ; polymorphonuclears, 66 per cent.; mononuclears, 34 per cent. He was killed at this time.

Necropsy.-The abdominal organs appeared to be congested, the liver and spleen being a dark red color. On opening the thorax, there was not a very evident lung lesion from the ventral surface. Complete examination, however, showed the greater part of the median and ventral lobes involved by a grayish pink lobular consolidation. Similar lesions were found along the main bronchi in the cephalic and caudal lobes, reaching the surface in very few places.

Microscopic: The lesion in the lungs consisted of a lobular polymorphonuclear alveolar exudate, in places becoming confluent. Small polymorphonuclear lesions at terminal bronchioles, similar to those described for Pig 21, were found in less involved regions. The bronchi contained considerable mucus with a few polymorphonuclear cells. The bronchial epithelium appeared normal. A few small areas of focal necrosis were found in the alveolar lesions, similar to those of Pig 25.

Bacteriologic: Lung cultures from the cephalic lobes gave numerous colonies of Bacillus bronchisepticus and a few pneumococcus colonies. Heart's blood cultures were negative.

The following protocol of Pig 27 has seemed to have special significance in the interpretation of the pathology of epidemic pneumonia, as it was an acute toxic case at the height of an animal epidemic, killed at a very early stage and thus demonstrates the primary type of reaction at the onset of toxic symptoms. 
PIG 27: History--April 18, 1919. The symptoms of illness first observed at noon were ill defined, a slight inactiveness and indisposition and a watery appearance of the eyes. The respirations were not increased, were quite shallow and apparently normal. The fur was not roughened. It was a very questionable illness. At 3 p. m. the symptoms were more distinct. The pig remained very quiet and the respirations were distinctly abnormal. They were of the short gasping type, not rapid, varying greatly at different times, between 80 and 150 per minute, but more often below 100 . There were occasional attacks of severe coughing during which the pig appeared to have great difficulty in breathing, as though suffocating. Between attacks shivering was noted. The temperature was $99.8 \mathrm{~F}$.; leukocyte count, 2,100 per c.cm.; polymorphonuclears, 10 per cent.; mononuclears, 85 per cent.; transitionals, 5 per cent. At this time the pig was killed by a blow on the back of the head. (Note: This method of killing was selected on account of not wishing to enter the factor of a respiratory anesthetic. It, however, does leave the possibility of nasal or pharyngeal hemorrhage which may be aspirated into the larger bronchi. Consequently, such hemorrhage found in the larger bronchi must be disregarded as a possible artefact. A better choice of means of killing might have been made. It is hardly possible that this would confuse in the interpretation of terminal bronchiolar and alveolar lesions since such a death means an immediate cessation of respirations.)

Necropsy.-In both cephalic and ventral lobes there was a grayish, lobular, semitranslucent consolidation along the main bronchi, reaching the surface in only a few places. The remaining peripheral part of these lobes was emphysematous or normal appearing. The medial lobes were completely involved in a fairly uniform gray consolidation. Both caudal lobes presented on the surface and cut section many small punctate hemorrhages, hardly noticeable as abnormal. These lobes were large and did not collapse. Figure 5 is a photograph of a section of the right caudal lobe, passing parallel to the main bronchus, and illustrates very well the above description. The dense hemorrhage in the main bronchus must be disregarded as a possible artefact.

Microscopic: Sections of the gray lobular consolidation along the main bronchi of the cephalic and ventral lobes showed this to be a rather diffuse polymorphonuclear alveolar exudate, yet preserving evidence of its primary localization in the terminal bronchioles, by the denser exudate in these regions and a partial atelectasis of the intervening alveoli. The terminal and smaller bronchioles commonly appeared dilated with a dense polymorphonuclear exudate: The widely spread small punctate hemorrhages of the large caudal lobes were found to be small areas of infundibular hemorrhage. The bronchus and terminal bronchioles leading to each of these areas were normal appearing, except for a distinct indication of a hyperactive secretion of mucus by the bronchial mucosa with numerous globules in the lumen and some darkly staining granules which could not with certainty be identified as bacteria. The hemorrhagic lesions, when seen at selected places cut parallel to a terminal bronchiole (Fig. 6), or followed in serial sections, were constantly found to begin at the site of transition of the columnar or cuboidal epithelium of the terminal bronchiole into the flat epithelium of the infundibular alveoli, and extend in triangular or treelike manner from this stem. This hemorrhage apparently effectually blocked the terminal bronchiole, extending a variable distance into it. The only other type of lesion in these caudal lobes was a replacement of the focal infundibular hemorrhages by polymorphonuclear cells, this occurring more frequenty in the left caudal lobe near the hilus, indicating a slightly older process in this region. Areas with partial replacement by polymorphonuclear cells were found, this taking place first in the immediate region of the terminal bronchiole and later in the periphery of the hemorrhagic lesion.

Bacteriologic: Lung cultures from both cephalic and caudal lobes gave abundant pure growth of the Bacillus bronchisepticus. Heart's blood cultures were negative. 
Guinea-pig 27 furnished very good material for the study of the mode of infection in this epidemic of pneumonia, since the caudal lobes presented widely distributed focal infundibular hemorrhagic lesions, representing a very early stage, and in one region showed the transition of these hemorrhagic lesions to a polymorphonuclear

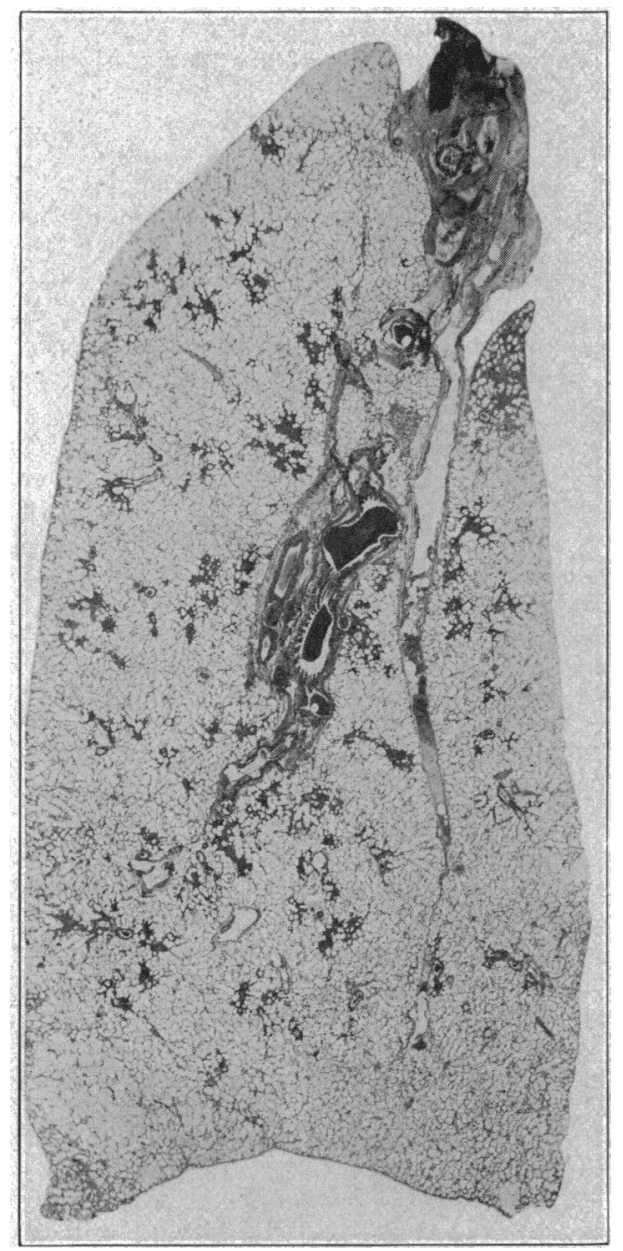

Fig. 5.-Microphotograph of a section of an entire caudal lung lobe of guineapig 27, showing the widespread infundibular hemorrhagic reaction during the early acute toxic stage of the pneumonia. Each small dark area represents an infundibular hemorrhage of the type illustrated by Figure 6 . There is no other lesion in this lobe.

type. The bronchi in general were normal appearing, except that the lumen most commonly was flooded with red blood cells. In a few bronchi only mucus globules were found, or polymorphonuclear 
leukocytes and mucus where the bronchus subtended a polymorphonuclear infundibular lesion.

Some indication of the mode of infection should be given by the location of the bacilli during the various stages of the primary focal lesion. For this study of bacteria in section Wolbach's modification of Giemsa's stain was found most useful. ${ }^{4}$ The $B$. bronchisepticus in tissue growth is very. small, comparable in size to the influenza bacillus. Previous writers have located the bacilli along the ciliated

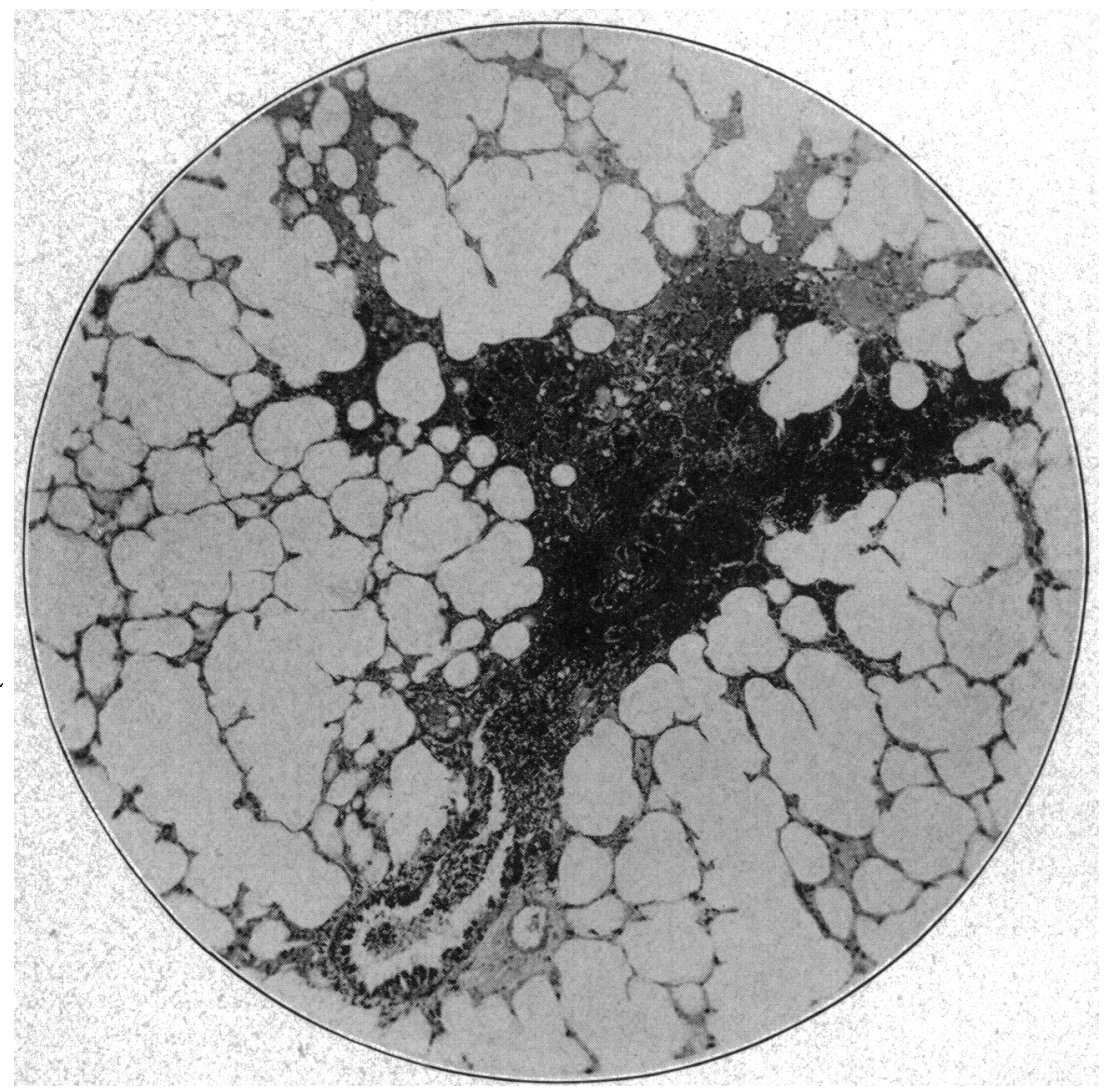

Fig. 6.-Microphotograph of a terminal bronchiole selected from the section illustrated by Figure 5, guinea-pig 27. The dark area consists of red blood cells, except that there are a few polymorphonuclear leukocytes in the immediate region of the terminal bronchiole and within the lumen.

border of the bronchial epithelium, but have not recognized the type of infundibular lesion presented by the entire caudal lobe of Pig 27, nor attempted correlation of the location of the bacilli with the stage of the infection.

4. Wolbach, S. B.: Studies on Rocky Mountain spotted fever, J. M. Res. 41: $1,1919$. 
The pathologic evidence presented by Pig 27 indicates that the infection descended the bronchi without producing a manifest lesion until it reached the thin pavement epithelium of the infundibuli, where focal hemorrhages resulted which flooded the smaller bronchi. Search for bacteria in these bronchi revealed a considerable number of very small deeply staining granules at the epithelial border, partly free but frequently appearing to adhere to globules of mucus which were being actively extruded from the epithelium. The location of the greater number of these granules was at the tips of the folds of the mucosa. They could be traced into the terminal bronchioles

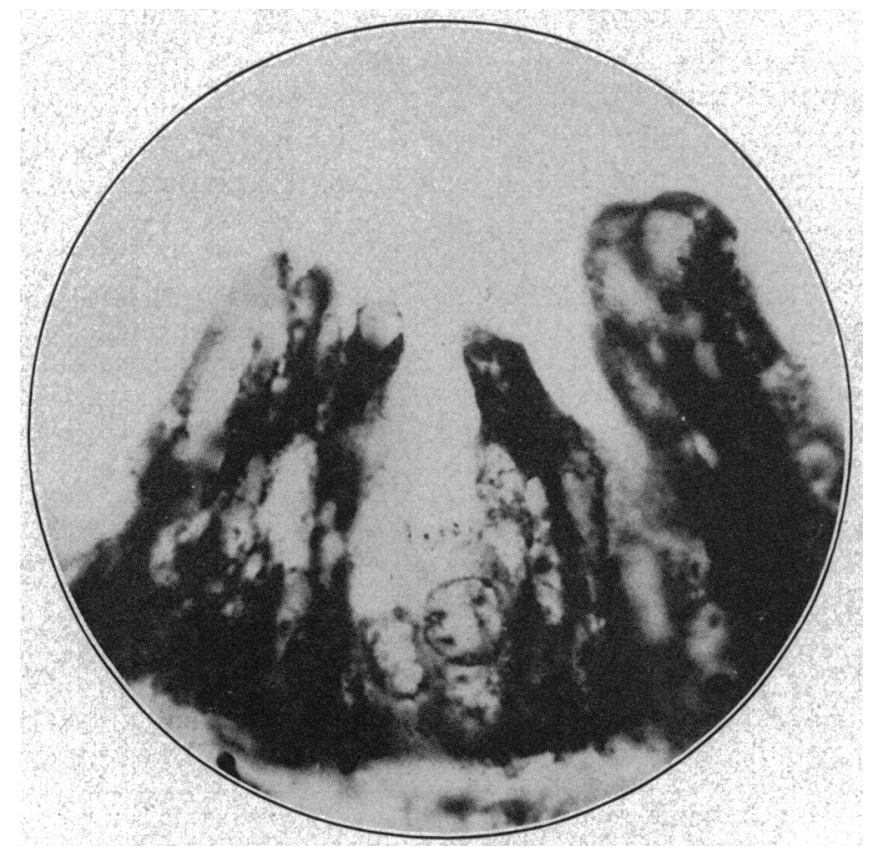

Fig. 7.-Microphotograph of bronchial mucosa from guinea-pig 27, to show the Bacillus bronchisepticus located between the folds of the mucosa. This area was selected from a small bronchus which subtended a polymorphonuclear infundibular lesion, similar to that illustrated by Figure 3. The bacilli are seen as small dots at the border of the epithelium.

and were found in the hemorrhagic exudates of the infundibuli. The identity of these granules is difficult to establish. The presence of the bacillus bronchisepticus in both caudal lobes was proven by culture, numerous colonies being obtained from a drop of lung juice withdrawn by capillary pipette through seared lung surface. The granules described were the only evidence of bacteria that could be found in relation to the earliest hemorrhagic infundibular lesion. 
The $B$. bronchisepticus was easily and certainly found in bronchi which subtended polymorphonuclear lesions. These bronchi usually contained mucus globules and cell detritus of degenerated polymorphonuclear leukocytes which most probably had descended from the infundibular lesion, as there was no evidence of a peribronchial reaction or the passing of leukocytes through the bronchial epithelium. The bacilli were found chiefly in the depressions between the folds of the mucosa (Fig. 7). They were quite numerous in places and situated in contact with the ciliated border of the epithelial cells. Rarely could bacilli be identified in the central bronchial exudate or in the infundibular lesion. In the larger bronchi at the hilus the bacilli were more uniformly distributed along the epithelial surface. The location of the bacilli between the folds of the mucosa in the smaller bronchi may represent the persistence of the infection only in the more protected regions near the infundibular lesion.

The following protocols, given in brief, will indicate the course of events in succeeding days of the epidemic:

Pig 28: History-April 18, 1919, an old breeding pig isolated on the preceding day on account of questionable illness. Leukocyte count, 10,700; polymorphonuclears, 66 per cent.; mononuclears, 34 per cent.; temperature, 101.6 F.; respirations 100 per minute. Killed by a blow on the head.

Necropsy.-Abdomen negative. Left cephalic lobe of the lungs completely involved by a gray type of consolidation. No hemorrhagic areas. Remaining lobes apparently normal except for marked anthracosis.

Microscopic: The involved lobe showed irregular areas of polymorphonuclear alveolar exudate, with intervening atelectasis or confluence of lesions. There also were areas of necrosis with abscess formation.

Bacteriologic: Cultures of the involved lobe gave a pneumococcus, pleural fluid and heart blood negative.

PIg 29: A young pig, weight $200 \mathrm{gm}$., found dead April 20.

Necropsy.-All lobes showed a dark red lobular involvement.

Microscopic: The lesion chiefly was that of capillary engorgement, centrally distributed with, in places, serous exudation or alveolar hemorrhage. One small bronchus was found dilated with polymorphonuclear cells and surrounded by polymorphonuclear alveolar exudate. Cultures from caudal lobes and trachea gave numerous colonies of the $B$. bronchisepticus.

PIg 30: History.-This pig was noted coughing April 20. He remained quiet but appeared active and irritable when disturbed. The respirations were not rapid, 100 per minute, but were of the short gasping type, irregular and interrupted by violent paroxysms of coughing as though suffocating. Shivering was noted. The temperature was $103 \mathrm{~F}$; leukocyte count, 14,000; polymorphonuclears, 23 per cent.; mononuclears, 77 per cent. He was killed by a blow on the head.

Necropsy.-The right cephalic, ventral and median lobes were completely involved in a moderately firm gray lobular consolidation. The right caudal lobe had a similar irregular area of gray consolidation at the hilus and along the main bronchus, but the remainder of the lobe was emphysematous and contained a few widely scattered punctate hemorrhages. All of the lobes of the left lung were emphysematous, did not collapse, and showed on the surface and cut section numerous small hemorrhagic spots, with no evident areas of gray involvement. The mucosa of the trachea and main bronchi was pale 
and normal appearing. These did not contain blood but a blood clot was found in one of the first stbdivisions of the left bronchus, subtending a small area of alveolar hemorrhage.

Microscopic: The gray lesions in the right lobes consisted of a dense polymorphonuclear infiltration in the infundibuli and surrounding alveoli, extending down into the dilated bronchioles. The intervening alveoli contained a few polymorphonuclear and mononuclear cells and appeared atelectatic. The peripheral part of the right caudal lobe and all lobes of the left lung, which showed rather uniformly distributed small hemorrhagic spots in gross, presented a type of lesion identical with that described in detail for the caudal lobes of Pig 27. These small hemorrhages were located in the infundibuli and the hemorrhagic exudate extended a variable distance down the bronchi. The mucosa of the bronchi was normal appearing except for evidence of active secretion of globules of mucus into the lumen. There was no dilatation of the bronchi or peribronchial reaction of any kind.

Bacteriologic: Cultures from the trachea and larger bronchi gave a heavy and pure growth of the B. bronchisepticus. Cultures from the blood cast in the left bronchus gave moderate numbers of the $B$. bronchisepticus. Culture from the right cephalic lobe of the lung gave a few colonies of the $B$. bronchiscpticus and culture from a hemorrhagic area of the left lung was negative. Examination of Giemsa stained sections for the location of the B. bronchiscpticus presented the same difficulties as in Pig 27. Numerous very small darkly stained granules were found near the folds of the mucosa, commonly adhering to globules of mucus, in the early hemorrhagic stage of the lesion. These could not positively be identified as bacteria. However, in the bronchi subtending the older polymorphonuclear lesions definite bacilli were found between the folds of the mucosa along the ciliated border of the cells.

PIG 31: Found dead April 21, several hours postmortem.

Vecropsy.-The lungs were found diffusely hemorrhagic, with evident laking of blood and marked empysema. There were no areas of gray consolidation.

Microscopic: Marked postmortem degeneration with desquamation of the bronchial epithelium. There appeared to be a diffuse capillary engorgement with central alveolar hemorrhage or serous exudation. Focal lesions could not be identified.

Bacteriologic: Cultures were overgrown by a spore-bearing contaminant.

PIG 32: History.-Observed ill April 28; respirations, 125 per minute, irregular and deep Pig quiet but active when disturbed. Leukocyte count, 15,500; polymorphonuclears, 10 per cent., mononuclears, 90 per cent. Killed by blow on head.

Necropsy.-There was a central lobular gray consolidation in all lobes with a few peripheral puncture hemorrages.

Microscopic: Material was lost in process of embedding.

Bacteriologic: Bacillus bronchisepticus recovered in pure culture from the right caudal and cephalic lobes.

PIg 33: History--May 5, had been under observation two days on account of inactiveness and abnormal respirations, 114 per minute, irregular and deep; leukocyte count, 17,700; polymophonuclears, 73 per cent., mononuclears, 27 per cent. Killed by blow on head.

Necropsy:-The cephalic and ventral lobes on both sides showed a lobular grayish red consolidation. Both caudal lobes were emphysematous and congested but with no distinct pneumonic foci.

Microscopic: The lesion in the cephalic and ventral lobes was a rather diffuse lobular polymorphonuclear alveolar exudate, most dense in the region of terminal bronchioles. Intervening uninvolved alveoli were atelectatic. A purulent pleurisy was found in section, not noted in gross. The larger bronchi contained no pus but a considerable amount of mucus. There was evidence of a 
previous healed infection in the lung, presented by dense accumulation of lymphoid cells rather widely distributed and having a relation either to blood vessels or obliteraed bronchi.

Bacteriologic: Lung cultures gave the B. bronchisepticus, heart blood culture negative.

PIG 40: History.-May 23, 1919, found dead, not noted ill on previous day, although cage was examined for sick pigs. He was one of twelve pigs that had been received a few days previously from outside stock. The entire lot had been kept separate in clean cages.

Necropsy-The lungs were very red and emphysematous. More extensive involvement in the central part of the lobes. The cut surface exuded blood stained fluid and air bubbles. No gray areas found.

Microscopic: Sections showed chiefly capillary engorgement, with serous or hemorrhagic alveolar exudate in the central region of the lobes.

Bacteriologic: Tracheal culture positive for the B. bronchisepticus, lung cultures negative.

PIG 48: This pig was a normal animal, killed May 17, and a lesion was found in the lungs which probably represents the final result in the healing of the focal infundibular lesions. Nothing was noted in gross but microscopic section revealed small foci of lymphoid cells distributed throughout the caudal lobes of the lung. These had no very constant relation to bronchi or blood vessels but occasionally were seen to be located at the termination of a bronchiole. Similar lesions were noted in Pig 33.

PIG 50: This pig was one which had gone through the epidemic without having developed evident illness. He was killed May 28, 1919. The lungs in gross appeared normal, except that the main bronchi appeared quite prominent in section. Microscopically these larger bronchi were found with a moderate peribronchial infiltration of lymphoid cells, a hyperplastic mucosa and an irregular lumen, evidence of a previous chronic bronchial infection with dilatation. Tracheal cultures gave a heavy growth of the hacillus bronchisepticus. Lung and heart blood cultures negative.

\section{BACTERIOLOGi}

This epidemic of pnetmonia in mice and guinea-pigs was studied chiefly from the standpoint of pathology, hence the bacteriologic data add little toward the establishment of the etiologic micro-organism of the disease. However, the $B$. bronchisepticus was recovered sufficiently often from the trachea and lungs to indicate that we were lealing with the same type of epidemic disease interpreted by other investigators to be caused by this bacillus.

The first identification of this micro-organism in relation to this cpidemic was in a mouse with the symptoms and pathology of purulent bronchitis. The infection among the mice had been noted several weeks previously but bacteriologic or pathologic observations were not made. The $B$. bronchisepticus in this and subsequent cases was identified by morphologic and cultural characteristics. The primary growth on blood agar plates was much less during the first twentyfour hours than at the end of forty-eight hours or in a twenty-fourlour subculture. The larger colonies were a grayish white color and emulsified readily in salt solution. Smears from the primary 
growth revealed a very small gram-negative bacillus, somewhat comparable to the influenza bacillus in size and morphology. In subcultures the size was considerably larger. These bacilli were actively motile in bouillon culture and gave a diffuse clouding. They grew readily on ordinary media. They did not ferment dextrose, lactose, galactose, saccharose, maltose, mannite, dextrin or inulin in Hiss' serum water media. They were distinctly aerobic and on potato slants gave a rich yellowish brown growth within forty-eight hours. All of these qualities are in agreement with the micro-organism described by Ferry, ${ }^{2}$ McGowan, ${ }^{5}$ Torry ${ }^{6}$ and Smith $^{7}$ as the etiologic factor in common epidemics of distemper and pneumonia in laboratory animals and called the Bacillus bronchisepticus.

table 1.-Epidemic Pneumonia in Mice. Necropsies.

\begin{tabular}{|c|c|c|c|}
\hline Date & Number & $\begin{array}{l}\text { Bacillus } \\
\text { bronchi- } \\
\text { septicus }\end{array}$ & 'Type of Pnewnonia \\
\hline $\begin{array}{l}3 / 27 / 19 \\
4 / 9 / 19 \\
4 / 9 / 19 \\
4 / 9 / 19 \\
4 / 11 / 19 \\
4 / 13 / 19 \\
4 / 13 / 19 \\
4 / 13 / 19 \\
4 / 18 / 19 \\
4 / 18 / 19 \\
4 / 18 / 19 \\
4 / 18 / 19 \\
4 / 18 / 19 \\
4 / 18 / 19 \\
4 / 24 / 19 \\
4 / 24 / 19 \\
4 / 27 / 19 \\
4 / 27 / 19 \\
4 / 27 / 19 \\
4 / 27 / 19 \\
6 / 1 / 19 \\
6 / 1 / 19 \\
6 / 1 / 19 \\
6 / 1 / 19\end{array}$ & $\begin{array}{r}1 \\
2 \\
3 \\
4 \\
5 \\
6 \\
7 \\
8 \\
9 \\
10 \\
11 \\
12 \\
13 \\
14 \\
15 \\
16 \\
17 \\
18 \\
19 \\
20 \\
21 \\
22 \\
23 \\
24\end{array}$ & $\begin{array}{l} \pm \\
+ \\
+ \\
\pm \\
= \\
= \\
\pm \\
\pm \\
= \\
- \\
= \\
\pm \\
\pm \\
= \\
+\end{array}$ & $\begin{array}{l}\text { Purulent bronchitis } \\
\text { Purulent bronchitis } \\
\text { Purulent bronehitis and lobular } \\
\text { Purulent bronchitis } \\
\text { Purulent bronchitis, lobar and hemorrhagic } \\
\text { Lobular and hemorrhagic } \\
\text { Hemorrhagic } \\
\text { Purulent bronchitis and hemorrhagic } \\
\text { Hemorrhagic } \\
\text { Lobular and hemorrhagic } \\
\text { Lobular and hemorrhagic } \\
\text { Purulent bronchitis and hemorrhagic } \\
\text { Hemorrhagic } \\
\text { Hemorrhagic and lobular } \\
\text { Hemorrhagic and purulent bronchitis } \\
\text { Purulent bronchitis } \\
\text { Negative } \\
\text { Hemorrhagic } \\
\text { Hemorrhagic and purulent bronehitis } \\
\text { Hemorrhagic } \\
\text { Lobular, hemorrhagic and purulent bronchitis } \\
\text { Lobular, hemorrhagic and purulent bronchitis } \\
\text { Lobular, hemorrhagic and purulent bronchitis } \\
\text { Purulent bronchitis and hemorrhagic }\end{array}$ \\
\hline
\end{tabular}

Lung cultures were made from twenty-four mice submitted to necropsy during the epidemic, with recovery of the $B$. bronchisepticus in only six of these, or 25 per cent. (Table 1). Tracheal cultures were not made from the mice which may partly account for the low percentage of positive cultures, since it is recognized that lung cultures are commonly negative in this disease when taken from the hemorrhagic lesions or healing processes. ${ }^{6}$

5. McGowan, J. P.: A laboratory epidemic of distemper, J. Path. \& Bacteriol. 15:372, 1910 .

6. Torrey, J. C., and Rahe, A. H.: Studies in canine distemper, J. M. Res. 17:291, 1913 .

7. Smith, Th.: Some bacteriologic and environmental factors in the pneumonias of lower animals, with special reference to the guinea-pig, J. M. Res., 24:291, 1914. 
The $B$. bronchisepticus was recovered more constantly from the guinea-pigs (Table 2). Fifteen pigs which developed symptoms of the epidemic disease were submitted to necropsy. Lung or tracheal cultures were made in fourteen of these, with recovery of the $B$. bronchisepticus in twelve. Of the two negative cultures, one contained only a pneumococcus and the other was overgrown by a spore bearing bacillus. The pneumococcus was recovered in three cases, each time from lung culture and in one of which there was a pneumococcus peritonitis.

TABle 2.-Epidemic Pneumonia in Guinea-Pigs. Necropsies.

\begin{tabular}{|c|c|c|c|c|c|c|}
\hline \multirow[t]{2}{*}{ Date } & \multirow[t]{2}{*}{ No. } & \multicolumn{2}{|c|}{$\begin{array}{c}\text { Bacillus } \\
\text { bronchisepticus }\end{array}$} & \multicolumn{2}{|c|}{ Pneumococcus } & \multirow[t]{2}{*}{ Type of Pneumonia } \\
\hline & & $\operatorname{Tr}$. & L. & Tr. & $\mathrm{L}$ & \\
\hline 4/ $6 / 19$ & 21 & + & + & - & - & $\begin{array}{l}\text { Polymorphonuclear and hemorrhagie } \\
\text { infundibular pneumonitis }\end{array}$ \\
\hline $\begin{array}{l}\text { 4/ } 7 / 19 \\
4 / 8 / 19\end{array}$ & 22 & $\because$ & $\ddot{+}$ & $\because$. & $\ddot{-}$ & $\begin{array}{l}\text { Hemorrhagic } \\
\text { Serohemorrhagic }\end{array}$ \\
\hline $4 / 15 / 19$ & 25 & .. & + & $\therefore$ & + & Lobular with necrosis \\
\hline $4 / 16 / 19$ & 26 & .. & + & $\because$ & $\dashv-$ & Infundibular, lobular, necrosis \\
\hline $4 / 18 / 19$ & 27 & $\therefore$ & t & $\therefore$ & - & Infundibular and lobular \\
\hline $4 / 18 / 19$ & 28 & . & - & .. & + & Lobular with necrosis \\
\hline $4 / 20 / 19$ & 29 & + & - & - & - & Serohemorrhagic \\
\hline $4 / 20 ! 19$ & 30 & + & + & - & - & Infundibular and lobular \\
\hline $4 / 21 / 19$ & 31 & . & .. & .. & .. & Serohemorrhagie \\
\hline $4 / 28 / 19$ & 32 & $\because$ & $\dddot{t}$ & $\therefore$ & $\ddot{-}$ & $\begin{array}{l}\text { Lobular } \\
\text { Lot }\end{array}$ \\
\hline 5) $5 / 19$ & 33 & + & $\therefore$ & $\because$ & .. & Lobular and healed infundibular \\
\hline 5) $5 / 19$ & 34 & + & . & - & .. & Lobular \\
\hline $5 / 13 / 19$ & 35 & + & $\therefore$ & -- & .. & Serohemorrhagie and lobular \\
\hline $5 / 23 / 19$ & 40 & + & .. & - & .. & Serohemorrhagie \\
\hline
\end{tabular}

Twenty apparently normal pigs were killed during the subsidence of the epidemic. Tracheal or lung cultures in these gave the $B$. bronchisepticus in eleven cases, frequently in abundant pure culture. A Type II pneumococcus was recovered from one pig.

The location of the bacilli in the tissues studied in microscopic section has been presented in detail in connection with the protocols, especially that of Pig 27.

\section{DISCUSSION}

The observations in this epidemic of pneumonia present two subjects for discussion. The first is the interpretation of the course and pathology, strictly confined to the animal epidemic. The other is the significance of these observations in the interpretation of human epidemics of pneumonia.

In this animal epidemic the infection apparently began as nasal infection or distemper among mice which were in very crowded quarters. Later it became transformed into a predominantly bronchial infection which in the more severe cases presented the symptoms and pathology of purulent bronchitis. The next step in the course of the epidemic was a change to a fulminating toxic pnemonia with 
very few respiratory symptoms and an emphysematous serohemorrhagic lung lesion. The subsidence was marked by a return of the predominant respiratory symptoms, but greatly varied lesions were found in the lungs, even of single animals, representing all stages from irregular areas of hemorrhage or polymorphonuclear exudate to purulent bronchitis or lobes with old obliterative bronchial lesions. The mortality rate was not very high among the mice and a large percentage probably became infected without being identified as ill. This was supported by almost invariably finding old lesions in the smaller lobes of supposedly normal mice killed late in the epidemic, and frequently finding active or even hemorrhagic lesions in mice that appeared quite well. A recurrent outbreak of the fulminating hemorrhagic pneumonia appeared in a few mice cages during the decline of the main epidemic, illustrating the possibility of a more virulent infection being superimposed on an existing or previous infection, as old lesions were found in conjunction with the hemorrhagic lesions in most of these mice.

The infection appeared among the guinea-pigs only after it had been prevalent among the mice several weeks. An environmental factor in the transmission of the infection is indicated by the fact that the first pigs affected were directly beneath the mice cages and the infection remained localized on this side of the animal room until an attempted cleaning spread normal appearing but evidently infected stock to other cages and pens. In the transmission of the infection from the mice to the guinea-pigs the stage of pneumonia appearing in the first pigs affected corresponded to that of the mice at that time. The distemper and the long period of chronic illness with marked respiratory embarrassment characteristic of purulent bronchitis did not occur in the pigs. The onset in the first pig affected was sudden and toxic.

The predominant lesion in this and several other pigs of the early nonfatal cases was a focal infundibular hemorrhage or polymorphonuclear exudate. This began at the point of transition from the cuboidal epithelium of the terminal bronchiole to the flat respiratory epithelium of the infundibulum. It extended a variable distance into the surrounding alveoli and appeared to discharge into the terminal bronchiole, effectually blocking this in many instances.

The most interesting and most completely studied example of this type of pneumonia was Pig 27 , killed at a very early stage when marked depression was evident. Both caudal lobes contained widely spread small infundibular hemorrhages. These focal hemorrhages would seem to represent the characteristic initial lesion of the early or milder cases at the beginning of the predominantly toxic stage of this epidemic of pneumonia. The location of this lesion, its evident 
older stage in the smaller more dependent lobes or along the main bronchi, and the generally normal appearing bronchi, would indicate a descending bronchial surface infection resulting in a toxic inflammatory reaction only when it reaches the thin respiratory epithelium of the infundibuli and thus comes in intimate contact with the blood capillaries. The first reaction, when there is sufficiently extensive involvement to produce systemic effects, is that of a severe toxemia, chill, prostration, slow irregular respiration and leukopenia. The second reaction is a respiratory embarrassment, due to the blocking of a sufficient number of terminal bronchioles to give symptoms of suffocation. The replacement of the primary hemorrhagic exudate with polymorphonuclear leukocytes is accompanied by a moderate blood leukocytosis. It is significant that all of the pigs presenting the focal infundibular pathology were killed. Those which died after several days illness presented confluent or lobular areas of polymorphonuclear exudate and those which died during the night without having been identified as ill in the previous day presented a more diffuse capillary engorgement, serous exudation and hemorrhage.

The pathology of these rapidly fatal cases was radically different from the infundibular pneumonitis occurring at the same time. This consisted of a more diffuse capillary lesion, most marked in the central or hilus portions of the lobes. It varied from a simple engorgement with slight serous exudation to capillary thrombosis, necrosis of the alveolar walls and hemorrhage into the alveoli. The relation of the bacteria to the more severe lesions indicated a diffuse hilus invasion probably by lymphatic channels but not clearly indicated. Older stages of this same type of lesion in more protracted cases seemed to be represented by irregular confluent areas of polymorphonuclear exudate and necrotic areas with bordering polymorphonuclear infiltration, representative of the places of capillary thrombosis. The difference between this type of pathology and the focal infundibular type could be explained by the increase of virulence of the invading micro-organism until it is enabled to invade diffusely through the bronchial mucosa and spread by lymphatic channels.

The subsidence of the epidemic among the guinea-pigs gave a great variety of lung lesions, as was observed among the mice Hemorrhagic lesions were superimposed on old obliterative lesions. In many apparently normal pigs killed after the epidemic had subsided old lung lesions were found, the commonest being numerous focal accumulations of lymphocytes throughout a lobe of the lung. These probably represented replacement of the focal infundibular polymorphonuclear lesions by mononuclear cells. A few cases were found with the characteristic lesion of healed purulent bronchitis. 
The predominant micro-organism recovered in lung and tracheal cultures was the Bacillus bronchisepticus, most commonly in pure culture but in a few cases complicated by a pneumococcus. The bacilli could not positively be identified in sections of lung during the earliest stage of infundibular hemorrhagic reaction, but numerous small granules located on the folds of the bronchial mucosa and adhering to mucus globules suggest strongly their identity as bacteria.

These observations on the course and pathology of an animal epidemic of pneumonia seem to be of significance in the interpretation of human epidemics of pneumonia. The recent epidemic of streptococcus pneumonia in the Army corps is the one most completely studied and best understood in this country. This epidemic most probably took its origin from the prevalent streptococcus pharyngitis and bronchitis which preceded the more severe pneumonia. ${ }^{8}$ The first stage of the epidemic of pneumonia was recognized and reported. ${ }^{6}$ It was characterized by a fairly uniform type of pathology, that of purulent bronchitis, and had a common association with measles. As the virulence of the streptococcus increased a more diffuse lobular pneumonia occurred more frequently and finally true epidemics of streptococus pneumonia occurred without any relation to measles. ${ }^{9}$ These epidemics developed rapidly, ran a course of six to eight weeks and the cases presented a predominantly toxic reaction with fulminating course and frequently a diffuse hemorrhagic lung lesion. During the subsidence of the epidemics a greater variety of pathology was encountered, with more cases of lobular pneumonia or interstitial bronchopneumonia as defined by MacCallum. Thus, if the entire course of the streptococcus infection is followed, it is found to have developed along a course very similar to the animal epidemic of pneumonia: from a prevalent mild pharyngeal and increasingly bronchial infection through a period of purulent bronchitis, to an independent epidemic of lobular and hemorrhagic pneumonia, with subsidence through somewhat similar stages in reverse order. This similarity with the course of events in the animal epidemic is significant of the life history of an epidemic of pneumonia.

8. Cole, R.: Etiology and clinical features of the pnetimonia occurring at a base hospital, J. A. M. A. 70:1146 (April 20) 1918. Irons, E. E., and Marine, D.: Streptococcal infection following measles and other diseases, J. A. M. A. 70:687 (March 9) 1918.

9. MacCallum, W. G.: Pathology of the epidemic streptococcal bronchopneumonia in the Army camps, J. A. M. A. 71:704 (Aug. 31) 1918. Miller, J. L., and Lusk, F. B.: Epidemic of streptococcus pneumonia and empyema at Camp Dodge, Iowa, J. A. M. A. 71:702 (Aug. 31) 1918. Lucke, B.: Postmortem findings in measles bronchopneumonia and other acute infections, J. A. M. A. 70:2006 (June 29) 1918. 
With this possible analogy in view it is most interesting to consider the pandemic of influenza and influenzal pneumonia in this light. The origin of the pandemic from all accounts seems to have been in Europe and most probably in the military camps, as the same environmental factors were present there as in the camps of this country where the epidemic of streptococcus pneumonia developed. A fact which immediately comes to the attention is that "there was prevalent during the late winter and early spring of 1916-1917, in the camps of northern France a severe type of bronchial infection, with a symptom complpex quite distinctive, very fatal, and which assumed such proportions by the end of January, 1917, as to constitute almost a small epidemic." ${ }^{10}$ This disease presented the typical picture of purulent bronchitis and the predominant micro-organism recovered from the lungs and bronchial exudate was the influenza bacillus, with the pneumococcus playing an important secondary rôle and only a comparatively rare hemolytic streptococcus. The investigators ${ }^{10}$ concluded that this disease was caused by the influenza bacillus and noted the fact that a mild influenza epidemic was actually in progress at that time.

This purulent bronchitis disappeared during the summer of 1917 but recurred during the winter of 1917-1918.11 The chief problem during that winter, however, was a disease or diseases commonly called "P. U. O," (pyrexia of unknown origin), claimed by many to be a capillary bronchitis and suggestive of an atypical influenza. ${ }^{12}$ Following this prevalence of “P. U. O." came in April and May, 1918, the first recognition of a widespread epidemic of influenza in the first and second English Armies in France. ${ }^{13}$ In July the pandemic quality and increasing severity of the infection were evident, ${ }^{\mathbf{1 4}}$ it reaching this country the latter part of August with the well known severe toxic symptoms of onset and hemorrhagic pulmonary complications. ${ }^{15}$ The preceding course of events suggests very strongly the gradual development of influenza in the camps of Europe through

10. Hammond, J. A. B., Rolland, W., and Shore, T. H. G.: Purulent bronchitis, Lancet 2:41, 1917. Abrahams, A., Hallows, N. F., Eyre, J. W. H., and French, H.: Purulent bronchitis. Its influenzal and pneumococcal bacteriology, Lancet 2:377, 1917.

11. McWalter, J. C.; The phenomena of purulent bronchitis, Brit. M. J. 1:119, 1918. Eyre, J. W. H., and Lowe, C. E. : Prophylactic vaccination against catarrhal affections of the respiratory tract, Lancet 2:485, 1918.

12. Pasteur, W., and Hudson, B.: A clinical contribution to the study of P. U. O., Lancet 1:95, 1918. Annotation: Camp infections, Lancet 1:810, 1918.

13. Influenzal Committee: The influenza epidemic in the British armies in France, 1918, Brit. M. J. 2:505, 1918.

14. Maude, A.: Influenza and purulent bronchitis, Lancet 2:324, 1918.

15. Keegan, J. J.: The prevailing pandemic of influenza, J. A. M. A. 71: 1051 (Sept. 28) 1918. 
a well defined stage of purulent bronchitis, a transition to the stage of infundibular pneumonitis (P. U. O.) and its final development into the stage of acute toxic hemorrhagic pneumonia.

This analogy might be extended further to include pneumococcus pneumonia, in which the well known pneumococcus bronchopneumonia of children would be representative of the developmental stage leading to the later acute toxic lobar pneumonia of young adults.

It is recognized that many features of epidemic pneumonia remain yet to be explained and established before acceptance should be given to the analogy presented in this paper. However, the recognition that epidemics have several stages in their development, each with certain distinctive features of symptomatology and pathology, should lead to a broader conception of epidemic diseases.

\section{SUMMARY}

1. This animal epidemic of pneumonia developed through the following stages, fairly well defined by symptoms and pathology:

(a) Nasopharyngeal infection (distemper).

(b) Mild bronchitis.

(c) Purulent bronchitis.

(d) Infundibular pneumonitis.

(e) Lobular pneumonia.

(f) Diffuse serohemorrhagic pneumonia.

(g) Subsidence in less distinct reverse order.

2. The epidemic of streptococcus pneumonia in the Army camps presented a somewhat similar course of events in its development.

3. The development of the pandemic of influenza can be traced in similar manner.

4. It is suggested that pneumococcus pneumonia should be considered in a similar course of development.

5. The recognition of purulent bronchitis as the natural precursor of an epidemic of acute toxic pneumonia places great significance on the prevalent influenza bacillus purulent bronchitis in the army camps of Europe during the winter of 1916-1917 and supports the view that the influenza bacillus is the cause of influenza. 